\title{
How to motivate participation and improve quality of crowdsourcing when building accessibility maps
}

\author{
Zhan Liu, Shaban Shabani, Nicole Glassey Balet, Maria Sokhn, Fabian Cretton \\ Institute of Information Systems \\ University of Applied Sciences and Arts Western Switzerland (HES-SO Valais-Wallis) \\ Sierre, Switzerland \\ \{zhan.liu,shaban.shabani,nicole.glassey,maria.sokhn,fabian.cretton\}@hevs.ch
}

\begin{abstract}
Crowdsourcing, as one of the most promising techniques for distributed problem-solving, requires sustained human involvement. Therefore, it also brings new challenges to data management, fundamentally data input and its quality. In this paper, we looked at various forms of user motivations and quality control of crowdsourcing when building accessibility maps mobile applications. We discuss how motivations could be used to contribute to our accessibility maps scenarios, and how data can be improved for two types of participants: individual participants and organization participants. We identified three useful techniques for improving data quality: qualification-based, reputation-based, and aggregation-based. In addition, based on our own mobile application (named WEMAP), we evaluated our approaches through focus group discussions and in-depth interviews.
\end{abstract}

Keywords-crowdsourcing, motivation, quality control, accessibility maps, wheelchair

\section{INTRODUCTION}

Accessibility-related mobile applications have become important and useful tools with which to help people with disabilities plan their travels. Maps-based accessibility solutions provide information about the available facilities of nearby urban infrastructures, and about transitory obstacles in the built environment. In fact, the demand for accessible map services is quite high. According to the UN Convention on Rights of Persons with Disabilities in 2007 [1], at least 650 million people live with disabilities worldwide, and disabilities affect $15-20 \%$ of every country's population on average. As for the European Union, it is estimated that there are 80 million disabled people, which means that at least one in four Europeans has a family member with a disability. And in Switzerland which had a population of 8.02 million in 2012, the number of people with disabilities has reached to 780,000 in 2012 [2].

Research has showed that one of the key reasons why disabled people do not frequently take vacations, for example, is that they often cannot travel on their own. They must take their families into consideration when deciding whether or not to go on vacation. However, the more assistance they need, the less the vacation would be family-oriented and contrarily, infrastructure-oriented. Thus, they need detailed and specific information in order to make a travel plan. Information is sometimes available online, however, in many cases, information is separated and sometimes inaccurate. Therefore, a high quality map service that gathers information about wheelchair accessibility is a real need.

One of the most popular contemporary techniques for spatial data collection, consumption, and evaluation, is crowdsourcing. This technique allows users to contribute wherever and whenever through the mobile devices, such as smart phones, smart watches, and tablets, and is already widely used in the domain of accessibility mapping [3]-[5]. Crowdsourcing has become a popular and successful techniques in the digital age, this mode of sourcing divided work between participants to achieve a cumulative result, which raises new possibilities. However, motivating user participation and ensuring the quality of crowdsourcing have become important challenges that we now face. These are also the most frequently asked questions during the progress of crowdsourcing work.

From a participants perspective, crowdsourcing can provide them with opportunities to increase exposure and working experiences, sharpen their creative skills, and improve perceived self-efficacy, as well as strengthen a sense of community [6]. In essence, crowdsourcing is defined as any sort of outsourcing that involves a large group of people actively participating in the project. The main subject of crowdsourcing is to collect the intelligences from a large number of participants. Therefore, coming to a better understanding of what motivates the crowd to participate in crowdsourcing competitions is of the greatest importance. [7] presented four key questions in a conceptual framework in order to identify fundamental dimensions and their relationships with crowdsourcing: 1) who is performing the task? 2) why are they doing it? 3) how is the task performed? 4) and what about the ownerships and what is being accomplished? These questions address the description of the performer of the task, the motivation of the participants and the incentives, and the approaches of the competition. We believe that another important dimension to be considered when exploring crowdsourcing is how motivational factors influence the quality of the outcomes.

Indeed, unclear crowdsourcing tasks with insufficient information and participants inattention can lead to participants providing low-quality contributions from the crowd [8]. The quality can get even worse in terms of creating accessibility maps applications for people with disabilities, because in this domain, details are often overlooked by the participants. For instance, many of us take several steps up and down 
curbs, or in and out of buildings, without even a second thought. However, for people who rely on wheelchairs to get around, even small steps can be considered as big travel difficulties and can cause huge problems. Addressing these issues requires fundamentally understanding the factors that impact quality, as well as quality-control approaches being used in crowdsourcing systems.

Therefore, in this study, we present technical approaches in order to explain how to motivate participation and to improve the quality of crowdsourcing when building accessibility maps mobile applications. We employed both focus group and individual interviews to evaluate the mobile application and to discuss our preliminary research results.

This paper is organized as follows: section 2 presents the background and related works, as well as business motivations. In section 3, we explain our approaches for motivating the participation of crowdsourcing and improving the quality of contribution. We report our preliminary results and evaluations in section 4. Finally, we conclude with a summary of the current work, and we present suggestions for future research.

\section{BACKGROUND AND RELATED WORK}

In this section, we first explore related works about motivation methodologies in the participation of crowdsourcing accessibility maps in the area of transportation for people with disabilities. Then we compare the existing technical solutions for quality control from crowdsourcing information.

\section{A. Motivations in crowdsourcing accessibility maps}

Motivation refers to the reasons that underlie behavior, which is characterized by willingness and volition [9]. It can be classified into two main types based on motivation orientation: intrinsic and extrinsic motivations. Intrinsic motivation remains an important construct, reflecting the natural human propensity to learn and assimilate. Intrinsic motivation can be regarded as the inherent satisfaction and enjoyment derived from an activity.

An experiment conducted by [10] showed that the factors of psychological empowerment could increase the intrinsic motivation to get work done more efficiently, including perceived self-efficacy [11], sense of community [12], and causal importance [13]. Authors found that understanding the social dynamics, increasing self-efficacy, and the participation of social work is crucial for the improvement of crowdsourcing in terms of crowd-mapping inaccessibility. Some research focused on crowdsourcing applied game theory, namely gamification [14]-[16], in order to map urban accessibility barriers and facilities. This method makes the task more engaging and interesting, and it may increase the level of motivation of its participants. In study [17] found that an important factor necessary to increase the intrinsic motivation is to frame a task as helping others. This finding is also validated by other studies. For instance, some applications focus on collecting information about the accessibility of points of interest [3], [5], and other applications aim to provide accessible paths [18], [19].
Another way in which to motivate users participation in accessibility crowdsourcing is through external motivation, which refers to the factors that bring value in return besides the task itself [20], [21]. For instance, prizes, monetary benefits, praise and recognition, and so on are considered to be extrinsic motivational factors. For example, Mechanical Turk is a paid service from Amazon which allows participants to contribute their work through an online crowdsourcing system [22]. There have been attempts to use Amazons Mechanical Turk platform to help collect data and to improve accessibility for the visually impaired. Another example from [23] describes the hiring of 123 distinct participants with monetary rewards to identify and rank sidewalk accessibility issues from a manually curated database of Google Street View images. In another study [24], user participation was incentivized with monetary prizes in order to establish a map for identifying and reporting the locations of places where cardiac patients can receive help, such as urgent care facilities.

These solutions could help to identify the factors of motivation in crowdsourcing accessibility. However, the lack of consideration of the different types and personal characteristics of participants could result in bias in the research results.

\section{B. Quality control in crowdsourcing accessibility systems}

When creating accessibility maps, some issues about the quality of collected information arise due to the inaccuracy of mobile sensors and the credibility of the users involved in the data gathering. Although any instance of crowdsourced data may be unreliable, various efforts have proposed models and techniques to quantitatively and objectively assess quality along different dimensions of an accessibility system, such as reliability, accuracy, relevancy, completeness, and consistency [25]. [26] proposed the report trustworthiness as a result of the type of source and its quality in terms of reliability. Differing from the common rating systems by expressing a value in a pre-fixed range, their trustworthiness assessment combined the accuracy of sensors, the source credibility of the crowd, and the authority of experts. [27] examined the effect of two quality control mechanisms on performance for accessibility problems: statistical filtering and multilevel review. By using these quality control methods, their accuracy rate increased from 88 percent to 93 percent. In recent studies, [10] validated that the increased psychological empowerment and self-efficacy led to improve quality of contribution. [28] focused on quality control in terms of finding bugs and errors based on patterns and rules extracted from the tracking data of users in OSM data, and they found that certain characteristics of user trajectories were directly linked to the type of feature.

However, participant profiles are often overlooked in quality control for creating accessibility maps. In this paper, we use selective recruitment and training of participants to provide the high-quality crowdsourcing data. 


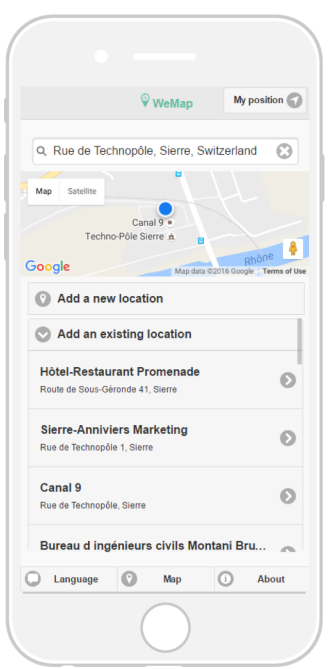

(a) searching POIs with a specific addresses.

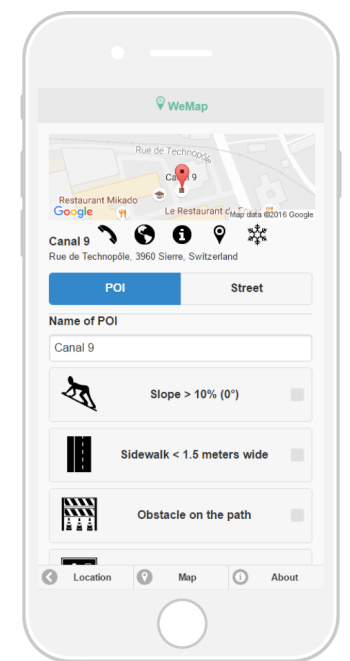

(b) add a new POI or a street situations.

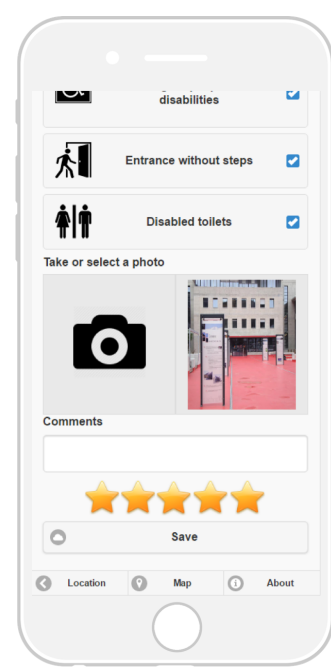

(c) review an existing POI.

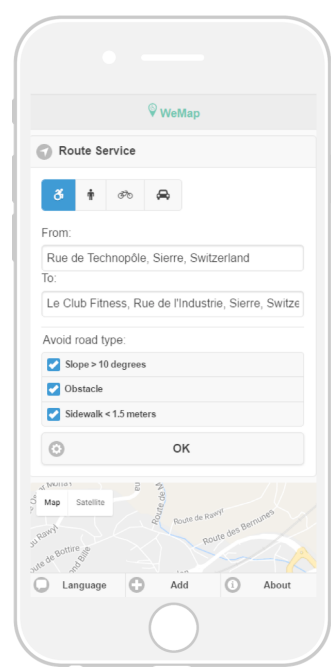

(d) route services form.

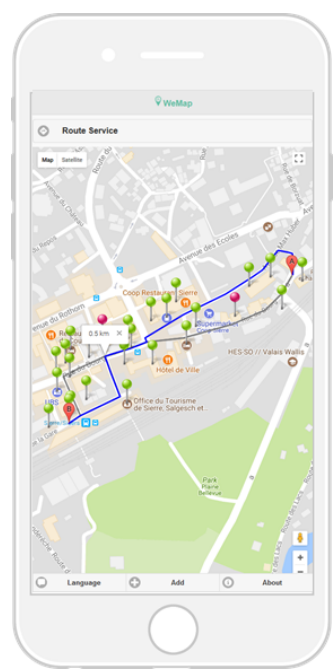

(e) route services generation and display.

Fig. 1: Mobile application user interfaces

\section{APPROACHES AND DESIGN PATTERNS FOR ACCESSIBILITY MAPS DEVELOPMENT}

In this section, we present in detail our approaches for increasing the motivation for contributions from crowd participants, as well as techniques for controlling the quality of participants. Table I summarizes the motivation and quality control aspect of our application. We have two target groups of participants: individual participants (i.e. single user) and organization participants (i.e. hotels and restaurants).

\section{TABLE I: APPROACHES FOR MOTIVATION AND QUAL- ITY CONTROL IN ACCESSIBILITY MAPS CROWD- SOURCING}

\begin{tabular}{|l|l|l|}
\hline Role & Motivation & Quality control \\
\hline $\begin{array}{l}\text { Individual } \\
\text { participants }\end{array}$ & $\begin{array}{l}\text {-Access to extra } \\
\text { services of the } \\
\text { application: e.g., } \\
\text { route services }\end{array}$ & $\begin{array}{c}\text {-Qualification tests } \\
\text {-Reputation system } \\
\text {-Aggregation techniques }\end{array}$ \\
\hline $\begin{array}{l}\text { Organization } \\
\text { participants }\end{array}$ & $\begin{array}{l}\text {-Delivery of certifi- } \\
\text { cates }\end{array}$ & $\begin{array}{l}\text {-Individual participants } \\
\text { ratings }\end{array}$ \\
\hline
\end{tabular}

\section{A. Boosting the motivation of participants}

Motivation in non-paid crowdsourcing scenarios is challenging, as participants expect a reward for their contributions. Therefore, in order to boost the participation and to motivate the participants to contribute to our accessibility map scenario, we provide limited access to the full version of the application, which additionally provides route services. Figure 1d and 1e shows the interface of the application for the route search aspect. After a certain number of route creations is reached, this service is disabled, and in order to re-gain access to the service, we ask participants to contribute by choosing one of the following two options:
- Review existing POIs - In this option, participants can choose whether to receive tasks related to their current location, i.e. POIs nearby, which introduces the spatial crowdsourcing technique [29], or any POIs provided by the application. The interface for assessing the existing POIs is shown in Figure 1c, and it includes visual information about parking places, slopes, obstacles, images, etc. Participants are asked to adjust any information that is not accurate, according to him/her. Later on, this information is used for the quality control aspect, in which we use techniques for maintaining the quality of the crowd participants.

- Insert new POI - This option allows participants to insert new data points, allowing them to choose either nearby locations or any point within a certain radius distance. Furthermore, users are able to search for specific addresses and places, a service which is not demonstrated by any other accessibility map applications (depicted in Figure 1a and $1 \mathrm{~b}$ ). The same interface as the reviewing aspect above is used, but with missing information, asking the participants to fill in the blank sections mentioned earlier.

We emphasize the importance of having accurate information about POIs that affect traveling for tourism. Therefore, we include tourism organizations and companies in our scenario, such as hotels and restaurants. Often, information that affects disabled people, such as parking places, entries and toilettes are difficult to find on online booking platforms, so it is important to have a service that can be shared among applications. As a motivational incentive, we make use of certificates for companies and public recognition for organizations. For instance, hotels and restaurants that are willing to contribute to our application by providing information about their organization are provided with a digital certificate that 


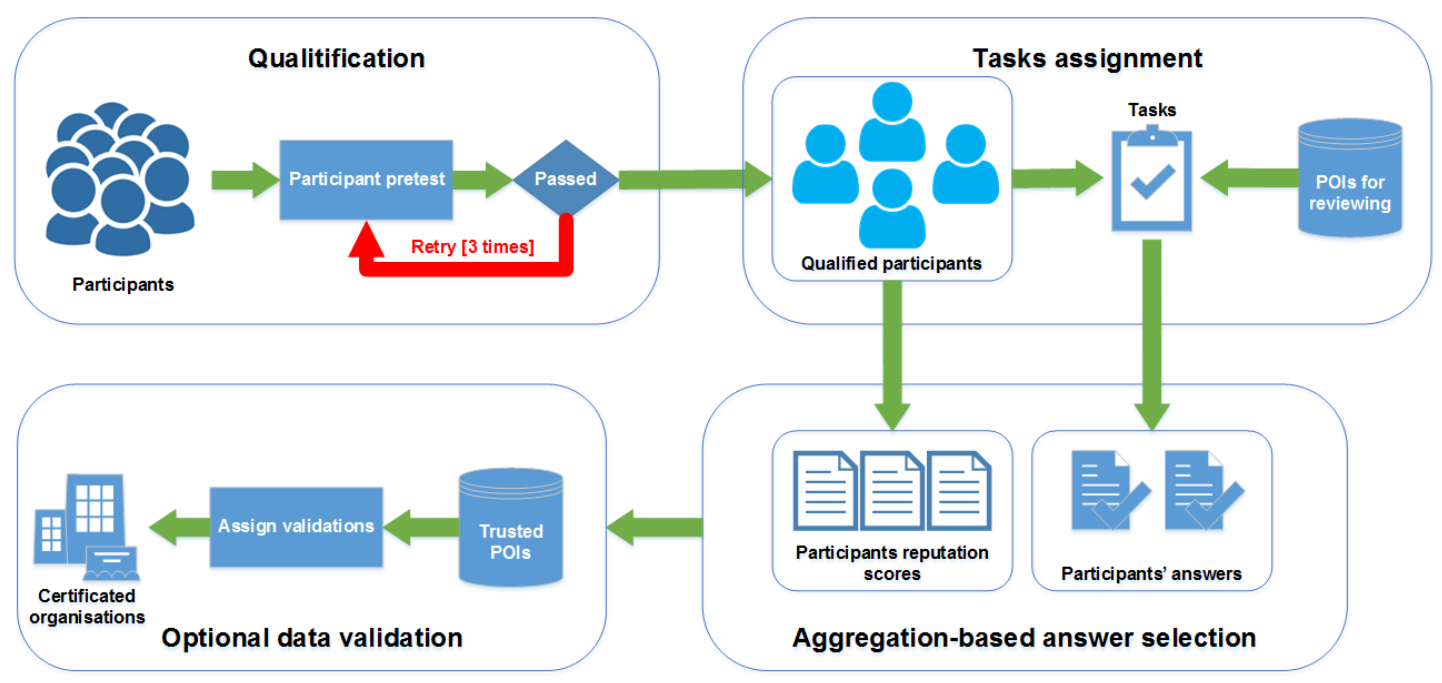

Fig. 2: Quality control methodology

they can then display on their website.

\section{B. Controlling the quality of crowdsourced data}

Quality control remains an open challenge in data crowdsourcing. Leaving a crowdsourcing application open for contribution to all participants can threaten the correctness of the overall results. It can that spam or lazy participants provide random labels, or even users that intentionally try to disrupt the system. On the other hand, low quality results might occur due to unknowledgeable workers. Hence, mechanisms are required to maintain the quality of data obtained by crowds. In our scenario, even though the application is open to any user, we apply three well-known quality control techniques including qualification, reputation, and aggregation-based techniques. The overall process is illustrated in Figure 2, followed by the description.

Qualification tests - Known as gold questions [30], this is a basic method with which to disqualify lazy workers and to educate workers that lack knowledge. The test contains reasonable questions, for which answers are known in advance. In our application, at the beginning, we ask users to review a few trivial tasks about POIs, which have visual information that corresponds to the information that the user is supposed to click on in the interface. For instance, we show POIs about a store which include an image of the store that shows that it is equipped with a parking place for disabled people. If the user answers the majority of questions correctly, he is allowed to further to use the application and continue to participate. Otherwise, after three failed attempts, he is excluded from participating any further.

Reputation system - This system relies on workers performance during crowdsourcing tasks [31]. Therefore, their contribution is weighted based on their scored points. In our scenario, reputation scores are initiated from the qualification tests. These scores are further used during the aggregation process. After each aggregation iteration - depending on the outcome of the voting participants reputation scores are updated, i.e. participants with wrong answers are penalized and their score is lowered, while the scores of those who answer correctly get increased.

Aggregation techniques - These are known as voting strategies, which repeat the same crowdsourcing task and aggregate their results [32]. Majority voting (MV) is a basic method that takes as its final output the answer with highest votes. In our application, we apply Weighted Majority Voting as a combination of MV with reputation scores, i.e. participants answers are weighted with their scores. Data being inserted by the participant is saved in a temporary database table, which has all new POIs that then must undergo the quality control check. As an example, we take a POI from this table and we ask five participants to review the information; for instance, whether or not a location has a parking place for disabled people. Each participants answer is multiplied with his current reputation score, and the answer with the highest score will be chosen. This information is inserted into the final database table, and becomes the final information which will be shown on the end-user interface. An example of the aggregation of the results is depicted in Figure 3.

Additionally, POIs data that relate to our registered participants from companies and organizations are further forwarded to them for a quality double check. If there is critical disagreement between the information provided by individual participants and organizations participants, manual checks are undertaken by the application support staff.

\section{PReliminary Results and EVAluation}

The WeMap mobile application was introduced in selected cities in Switzerland. Presently, almost 900 collections were added into our database, including the POIs (point of interests) and obstacles on the street. In order to evaluate the feasibility of our approach, we conducted four focus group interviews with sixteen participants from different age ranges and backgrounds (as shown in the table II), as well as four in-depth individual interviews from four tourism experts in 


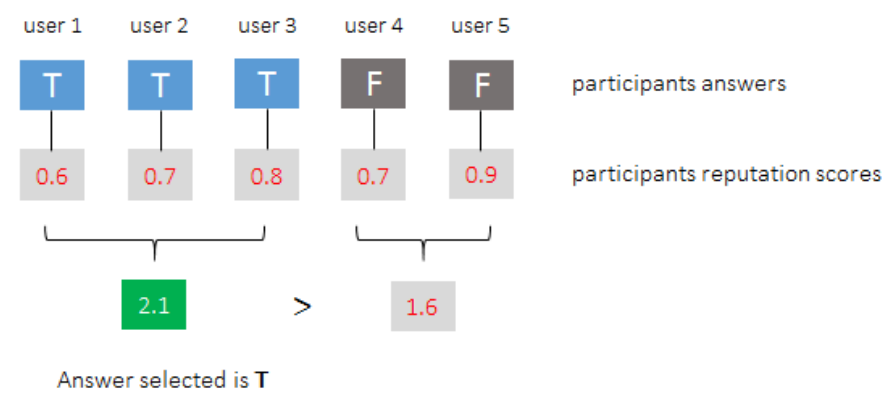

Fig. 3: Aggregation phase of the participants answers

four different cities in Switzerland. During the focus group interviews, we asked questions about the motivations for using this application. Most affirmed that this application was useful to get accessible information and the map-based route services can make travel easier. They expressed that they were willing to use this application, and the reasons were reflected in both intrinsic and extrinsic aspects. The main intrinsic motivations were the sense of community (e.g. to help people, social psychology), and self-efficacy (e.g. the beliefs of power to affect situations, ability to accomplish a task). The external motivations were mainly reflected in the acquisition of the full version of the application. Another important aspect highlighted by the results was related to business participants, mostly service providers, such as hotels, restaurants, and other organizations. They aim to provide quality services for people with disabilities, in addition to establishing and enhancing the brand image. We also noticed that the participants include not only wheelchair users and their families or friends, but also parents with baby strollers, for example.

\section{TABLE II: DEMOGRAPHIC DATA ON FOCUS GROUP PARTICIPANTS}

\begin{tabular}{|l|l|c|c|}
\hline & Mean (std.) & Range & Percentage \\
\hline \multirow{2}{*}{ Gender } & - & Male & $56 \%$ \\
& & Female & $44 \%$ \\
\hline \multirow{4}{*}{ Age } & \multirow{3}{*}{$38.94(15.06)$} & $<25$ & $19 \%$ \\
& & $25-40$ & $39 \%$ \\
& & $51-50$ & $19 \%$ \\
& & $>60$ & $12 \%$ \\
& & & $12 \%$ \\
\hline
\end{tabular}

To evaluate the quality of the crowdsourcing, we invited four tourism experts who have strong knowledge of local facilities in order to compare the results between the qualified participants (i.e. selected skilled participants) and non-qualified participants (i.e. normal participants) through our selective quality control methodology. The samples were based on the information collected from 240 POIs (120 POIs were collected from qualified participants and 120 POIs were collected from non-qualified participants). We found that the accuracy rate of the accessibility map crowdsourcing increased $12 \%$, from $83 \%$ to $95 \%$. Before the interviews, the tourism experts were asked to control the quality of crowdsourcing information from three parts, which include accessibility criteria (e.g. parking for disabled people, scope, etc.), photos, and comments from participants. During the interviews, they were asked to discuss the problems of the participants. These problems can be summarized into four categories: 1) some accessibility criteria were misappropriated, such as the slope degree and the width of sidewalk; 2) service information, such as the disabled toilet availability and the parking information, is often missing; 3 ) the photo did not conform to the selected criteria; and 4) some accessibility information was incomplete. For example, some obstacles on the path were only temporary; however no accurate information about the duration of the obstacle was provided.

\section{CONClusion}

In this paper, we discuss how to motivate participation and improve the quality of crowdsourcing when building accessibility maps. We compare the existing methods of motivation and quality control in crowdsourcing, and explain their deficiencies. Most importantly, we present the rolebased approaches and design patterns for accessibility maps mobile applications. Our approaches focus on both individual participants and organization participants, and we explain each of them in more detail with users interfaces. To evaluate the feasibility of approaches and the usability of applications, we conducted focus group and individual interviews, respectively. The results highlight that the accuracy rate has a significant increase after applying the intervention of quality control methods. Finally, we summarize the quality issues when using an accessibility maps application. Future research should concentrate on testing our approaches to expand a more accurate evaluation and to valid in more areas. Moreover, the comparison of the accuracy rate for unpaid and paid scenarios should be also interesting to test.

\section{ACKNOWLEDGMENT}

The work described in this paper is supported by the University of Applied Sciences and Arts Western Switzerland (HESSO) under grant number 61932. We thank all participants for using and evaluating our mobile application. We especially thank Dominique Rion from Swiss Post, and other experts who supported us in the data collection and quality evaluation.

\section{REFERENCES}

[1] "Convention on the rights of persons with disabilities," https://www.un.org/development/desa/disabilities/convention-on-therights-of-persons-with-disabilities.html, accessed: 2017-09-10.

[2] "Swiss federal statistical office," https://www.bfs.admin.ch/bfs/en/home.html, accessed: 2017-09-10.

[3] "Wheelmap," http://wheelmap.org/en/ , accessed: 2017-09-10.

[4] Z. Liu, N. G. Balet, M. Sokhn, and E. De Gaspari, "Crowdsourcingbased Mobile Application for Wheelchair Accessibility," 2017. [Online]. Available: http://scholarworks.calstate.edu/handle/10211.3/190198

[5] “Axsmap," http://www.axsmap.com/, accessed: 2017-09-10.

[6] P. Trompette, V. Chanal, and C. Pelissier, "Crowdsourcing as a way to access external knowledge for innovation," Jul. 2008. [Online]. Available: https://halshs.archives-ouvertes.fr/halshs-00367373/document

[7] Y. Zhao and Q. Zhu, "Evaluation on crowdsourcing research: Current status and future direction," Information Systems Frontiers, vol. 16, no. 3, pp. 417-434, Jul. 2014. [Online]. Available: https://link.springer.com/article/10.1007/s10796-012-9350-4 
[8] J. J. Chen, N. J. Menezes, A. D. Bradley, and T. A. North, "Opportunities for crowdsourcing research on amazon mechanical turk," in CHI 2011 Workshop Crowdsourcing and Human Computation, Oct. 2011. [Online]. Available: http://crowdresearch.org/chi2011workshop/papers/chen-jenny.pdf

[9] E. R. Lai, "Motivation: A literature review," Person Researchs Report, 2011.

[10] J. Goncalves, S. Hosio, J. Rogstadius, E. Karapanos, and V. Kostakos, "Motivating participation and improving quality of contribution in ubiquitous crowdsourcing," Computer Networks, vol. 90, pp. 34-48, Oct. 2015. [Online]. Available: http://www.sciencedirect.com/science/article/pii/S1389128615002224

[11] M. L. Ohmer, "Citizen Participation in Neighborhood Organizations and Its Relationship to Volunteers' Self- and Collective Efficacy and Sense of Community," Social Work Research, vol. 31, no. 2, pp. 109-120, 2007. [Online]. Available: http://www.jstor.org/stable/42659652

[12] M. Prezza, M. Amici, T. Roberti, and G. Tedeschi, "Sense of community referred to the whole town: Its relations with neighboring, loneliness, life satisfaction, and area of residence," Journal of Community Psychology, vol. 29, no. 1, pp. 29-52, Jan. 2001. [Online]. Available: http://onlinelibrary.wiley.com/doi/10.1002/15206629(200101)29:1;29::AID-JCOP3;3.0.CO;2-C/abstract

[13] C. Peterson, "Personal control and well-being," in Well-being: Foundations of hedonic psychology, D. Kahneman, E. Diener, and N. Schwarz, Eds. New York: New York: Russell Sage, 1999, pp. 288-30

[14] P. Salomoni, C. Prandi, M. Roccetti, V. Nisi, and N. J. Nunes, "Crowdsourcing Urban Accessibility:: Some Preliminary Experiences with Results," in Proceedings of the 11th Biannual Conference on Italian SIGCHI Chapter, ser. CHItaly 2015. New York, NY, USA: ACM, 2015, pp. 130-133. [Online]. Available: http://doi.acm.org/10.1145/2808435.2808443

[15] L. von Ahn, S. Ginosar, M. Kedia, R. Liu, and M. Blum, "Improving Accessibility of the Web with a Computer Game," in Proceedings of the SIGCHI Conference on Human Factors in Computing Systems, ser CHI '06. New York, NY, USA: ACM, 2006, pp. 79-82. [Online]. Available: http://doi.acm.org/10.1145/1124772.1124785

[16] C. Prandi, V. Nisi, P. Salomoni, and N. J. Nunes, "From Gamification to Pervasive Game in Mapping Urban Accessibility," in Proceedings of the 11th Biannual Conference on Italian SIGCHI Chapter, ser. CHItaly 2015. New York, NY, USA: ACM, 2015, pp. 126-129. [Online]. Available: http://doi.acm.org/10.1145/2808435.2808449

[17] J. Rogstadius, V. Kostakos, A. Kittur, B. Smus, J. Laredo, and M. Vukovic, "An assessment of intrinsic and extrinsic motivation on task performance in crowdsourcing markets," in International AAAI Conference on Web and Social Media, ser. ICWSM, 2011, Conference Paper, pp. 321-328. [Online]. Available: http://people.eng.unimelb.edu.au/vkostakos/files/papers/icwsm11.pdf

[18] E. L. Hillsman and S. J. Barbeau, "Enabling Cost-Effective Multimodal Trip Planners through Open Transit Data National Center for Transit Research.” [Online]. Available: https://www.nctr.usf.edu/2011/05/enablingcost-effective-multimodal-trip-planners-through-open-transit-data-2/

[19] M. Roccetti, G. Marfia, and C. E. Palazzi, "Entertainment beyond divertissment: using computer games for city road accessibility," Computers in Entertainment (CIE), vol. 9, no. 2, p. 10, 2011.

[20] R. M. Ryan and E. L. Deci, "Intrinsic and Extrinsic Motivations: Classic Definitions and New Directions," Contemporary Educational Psychology, vol. 25, no. 1, pp. 54-67, Jan. 2000. [Online]. Available: http://www.sciencedirect.com/science/article/pii/S0361476X99910202

[21] G. Paolacci, J. Chandler, and P. G. Ipeirotis, "Running Experiments on Amazon Mechanical Turk," Social Science Research Network, Rochester, NY, SSRN Scholarly Paper ID 1626226, 2010. [Online]. Available: https://papers. ssrn.com/abstract $=1626226$

[22] J. Ross, L. Irani, M. S. Silberman, A. Zaldivar, and B. Tomlinson, "Who Are the Crowdworkers?: Shifting Demographics in Mechanical Turk," in CHI '10 Extended Abstracts on Human Factors in Computing Systems, ser. CHI EA '10. New York, NY, USA: ACM, 2010, pp. 2863-2872. [Online]. Available: http://doi.acm.org/10.1145/1753846.1753873

[23] K. Hara, V. Le, and J. Froehlich, "A Feasibility Study of Crowdsourcing and Google Street View to Determine Sidewalk Accessibility," in Proceedings of the 14th International ACM SIGACCESS Conference on Computers and Accessibility, ser. ASSETS '12. New York, NY, USA: ACM, 2012, pp. 273-274. [Online]. Available: http://doi.acm.org/10.1145/2384916.2384989
[24] R. M. Merchant, D. A. Asch, J. C. Hershey, H. M. Griffis, S. Hill, O. Saynisch, A. C. Leung, J. M. Asch, K. Lozada, L. D. Nadkarni, A. Kilaru, C. C. Branas, E. M. Stone, L. Star, F. Shofer, G. Nichol, and L. B. Becker, "A crowdsourcing innovation challenge to locate and map automated external defibrillators," Circulation. Cardiovascular Quality and Outcomes, vol. 6, no. 2, pp. 229-236, Mar. 2013.

[25] E. Agichtein, C. Castillo, D. Donato, A. Gionis, and G. Mishne, "Finding High-quality Content in Social Media," in Proceedings of the 2008 International Conference on Web Search and Data Mining, ser. WSDM '08. New York, NY, USA: ACM, 2008, pp. 183-194. [Online]. Available: http://doi.acm.org/10.1145/1341531.1341557

[26] C. Prandi, S. Mirri, and P. Salomoni, "Trustworthiness Assessment in Mapping Urban Accessibility via Sensing and Crowdsourcing," in Proceedings of the First International Conference on IoT in Urban Space, ser. URB-IOT '14. ICST, Brussels, Belgium, Belgium: ICST (Institute for Computer Sciences, Social-Informatics and Telecommunications Engineering), 2014, pp. 108-110. [Online]. Available: http://dx.doi.org/10.4108/icst.urb-iot.2014.257267

[27] K. Hara, V. Le, and J. Froehlich, "Combining Crowdsourcing and Google Street View to Identify Street-level Accessibility Problems," in Proceedings of the SIGCHI Conference on Human Factors in Computing Systems, ser. CHI '13. New York, NY, USA: ACM, 2013, pp. 631-640. [Online]. Available: http://doi.acm.org/10.1145/2470654.2470744

[28] A. Basiri, M. Jackson, P. Amirian, A. Pourabdollah, M. Sester, A. Winstanley, T. Moore, and L. Zhang, "Quality assessment of OpenStreetMap data using trajectory mining," Geo-spatial Information Science, vol. 19, no. 1, pp. 56-68, Jan. 2016. [Online]. Available: http://dx.doi.org/10.1080/10095020.2016.1151213

[29] Z. Chen, R. Fu, Z. Zhao, Z. Liu, L. Xia, L. Chen, P. Cheng, C. C. Cao, Y. Tong, and C. J. Zhang, "gMission: A General Spatial Crowdsourcing Platform," Proc. VLDB Endow., vol. 7, no. 13, pp. 1629-1632, Aug. 2014. [Online]. Available: http://dx.doi.org/10.14778/2733004.2733047

[30] D. Oleson, A. Sorokin, G. Laughlin, V. Hester, J. Le, and L. Biewald, "Programmatic Gold: Targeted and Scalable Quality Assurance in Crowdsourcing," in Workshops at the Twenty-Fifth AAAI Conference on Artificial Intelligence, Aug. 2011. [Online]. Available: https://www.aaai.org/ocs/index.php/WS/AAAIW11/paper/view/3995

[31] M. Allahbakhsh, A. Ignjatovic, B. Benatallah, S. M. R. Beheshti, E. Bertino, and N. Foo, "Reputation management in crowdsourcing systems," in 8th International Conference on Collaborative Computing: Networking, Applications and Worksharing (CollaborateCom), Oct. 2012, pp. 664-671.

[32] C. C. Cao, J. She, Y. Tong, and L. Chen, "Whom to ask?: jury selection for decision making tasks on micro-blog services," Proceedings of the VLDB Endowment, vol. 5, no. 11, pp. 1495-1506, 2012. 